\title{
Solving the Multistage PMU Placement Problem by Integer Programming and Equivalent Network Design Model
}

\author{
Ou Sun · Neng Fan
}

May 25, 2018

\begin{abstract}
Recently, phasor measurement units (PMUs) are becoming widely used to measure the electrical waves on a power grid to determine the health of the system. Because of high expense for PMUs, it is important to place minimized number of PMUs on power grids without losing the function of maintaining system observability. In practice, with a budget limitation at each time point, the PMUs are placed in a multistage framework spanning in a long-term period, and the proposed multistage PMU placement problem is to find the placement strategies. Within each stage for some time point, the PMUs should be placed to maximize the observability and the complete observability should be ensured in the planned last stage. In this paper, the multistage PMU placement problem is formulated by a mixed integer program (MIP) with consideration of the zero-injection bus property in power systems. To improve the computational efficiency, another MIP, based on the equivalent network flow model for the PMU placement problem, is proposed. Numerical experiments on several test cases are performed to compare the two MIPs.
\end{abstract}

Keywords PMU Placement · Multistage Placement · Zero-Injection Buses · Integer Programming $\cdot$ Network Design

\section{Introduction}

As part of the Supervisory Control And Data Acquisition system for situational awareness and wide-area monitoring of the electric power systems, a device called Phasor Measurement Unit (PMU) measures several state variables, and multiple PMUs distributed throughout the power system can form a phasor network for collecting the information. There were fewer than 500 research-grade PMUs installed in North American power grid in 2009, while by March 2015, there are almost 2000

O. Sun · N. Fan $(\bowtie)$

Department of Systems and Industrial Engineering, University of Arizona, Tucson, AZ 85721, USA.

E-mail: nfan@email.arizona.edu 
PMUs (see [1]). The number grows fast recently because hundreds of million dollars have been invested in PMU technology.

Because of high cost of PMUs and nonexistence of communication facilities in some substations, it is important to minimize their number of placement without losing the ability to monitor the entire power system. An undirected graph $G=(V, E)$, called a Power System Graph, is usually applied to model a power system, where the vertex set $V$ represents a set of buses, and an edge $\left(v_{i}, v_{j}\right) \in E$ represents a transmission line joining two buses $v_{i}, v_{j} \in V$. A PMU at a bus measures the voltage of a bus and the current of each line incident to this bus. These buses and lines are said to be observed.

The optimal PMU Placement Problem (PPP) is to find the minimum number of PMUs placed on buses such that all buses and lines can be observed. Some integer programming (IP) approaches have been proposed for solving the PPP [2-13], while some heuristic methods, such as genetic algorithms, Tabu search, simulated annealing, and particle swarm optimization, were also proposed in [7, 14, 15]. Among these IP approaches, some of them (e.g., [9-13]) considered the contingencies such as loss of a single line or PMU, while some of them (e.g., [3, 4, 7, 9]) included the conventional flow measurements. In $[5,12,16]$, PMUs were classified by the number of channels that it can observe. The PPP is intuitively related to some classic combinatorial optimization problems, such as the dominating set problem (DSP), which requires all vertices to be observed (see [17-19]), and the vertex cover problem (VCP) which requires all edges to be observed (see [20,21]). However, the existence of Ohm's law and Kirchhoff's current law (or zero-injection property) in power systems, distinguishes the PPP from them. In [6,22], the term power dominating set problem was proposed because of the relationship between the PPP and the DSP. Besides the proof of the NP-hardness of the PPP, the special version of this problem on graphs with structures of trees was also studied in [22]. Thereafter, many papers have studied the power dominating set problem on some special graphs, such as planar graphs [23], product graphs [24], cylinders, tori, generalized Petersen graphs [25, 26], etc. For the PPP, its different versions, such as minimizing placed PMUs and maximizing observability, are also proved to be NP-hard [22,27].

The PPP is to find a placement for complete observability, which means every bus and every line are observed. However, because of the expensive cost for installation of PMUs and budget limitation in practice, placing PMUs in a large power system to ensure complete observability at one time point is not realistic [28]. Therefore, instead of complete observability at a single stage, a multistage model for PMU placement should be proposed for an implementation schedule and the ultimate goal is to ensure complete observability at the planned last stage. Here, a stage means a time point. Selecting the buses to place PMUs at each stage should gradually increase the overall observability. Therefore, for a given number of PMUs (limited by the budget) at each stage, the objective is to maximize the observability by selecting the buses to place PMUs. Thus, the stages before last stage all have incomplete observability while the last stage will guarantee the complete observability. This is the proposed Multistage PMU Placement Problem (MPPP), and there are some studies for this problem in [8,29-34]. However, methods in [29,30] were based on greedy or random selection of buses at each stage, and were more of analytic approaches. Thus, 
they cannot be extended to large graphs to ensure optimality. In [8,31], although IP approaches were used, none of them considered the property of zero-injection buses, which can reduce the number of total PMUs. Even though this property is considered in $[33,34]$, it lacks consideration of redundant observation times which can help to improve the robustness of the system (see [33]), or only approximate solutions can be achieved by a meta-heuristic algorithm (see [34]). In summary, the exact solution approaches are not clearly studied with consideration of the zero-injection bus property in the literature, and the computational efficiency is always a burden for large scale power systems.

In this paper, we solve the multistage PMU placement problem with the exact solution approaches, and make the following contributions: (i) A mixed integer program (MIP) is proposed to formulate the MPPP, with consideration of both zero-injection bus property and maximum bus observability at each stage; (ii) The redundant observations for each bus can be achieved as a benefit of maximum observability at each stage; (iii) To improve the computational efficiency, we extend the idea of an augmented network for solving the single-stage PPP to solve the MPPP. An equivalent network design model for the MPPP is proposed based on the augmented network and it utilizes the network flow structure which greatly reduce the computational complexity.

The remainder of this paper is organized as follows: In Section 2, the IP formulation for PPP is reviewed after introduction of the Ohm's law and Kirchhoff's current law in power systems, and then the IP formulation for MPPP is presented. Additionally, some techniques for linearizing the nonlinear terms in the IP formulations are applied. In Section 3, we introduce an augmented network flow model for PPP with the consideration of zero-injection bus property. Based on this model, the MPPP is equivalent to a network design problem, and the IP formulation for MPPP is proposed. Section 4 performs the numerical experiments on several test systems, while Section 5 concludes the paper.

\section{IP Formulations for PMU Placement Problem}

\subsection{Bus observability and IP formulation for PMU placement problem}

As introduced in Section 1, the PPP can be viewed as a special kind of the DSP with additional consideration of the zero-injection bus property. The DSP ensure that each vertex is covered at least once. In the PPP, if all buses are observed, all lines will be observed by the Ohm's law (as explained below). Thus, if we solve the DSP within a corresponding power system graph, the minimum number of vertices in the dominating set of the DSP provides an upper for the minimum number of PMUs. In some papers $([2-4,8])$, when the property of zero-injection buses are not considered, they actually solved the DSP.

In the PSG $G=(V, E)$ to model a power system, some parameters of this graph are given:

- Matrix $A=\left(a_{i j}\right)_{|V| \times|V|}$ : neighborhood matrix. Let $a_{i j}$ 's be values such that $a_{i j}=1$ if $i=j$ or buses $v_{i}, v_{j}$ are connected by a transmission line, and $a_{i j}=0$ otherwise. 
- $N\left(v_{i}\right)=\left\{v_{j} \in V \mid\left(v_{i}, v_{j}\right) \in E\right\}, N\left[v_{i}\right]=N\left(v_{i}\right) \cup\left\{v_{i}\right\}:$ sets of open/closed neighborhood of vertex $v_{i}$. Let $d[i]$ be the cardinality of closed neighborhood of a bus $v_{i}$, i.e., $d[i]=\left|N\left[v_{i}\right]\right|$.

- Zero-injection buses: $Z_{i}=1$ indicates that vertex $v_{i}$ is a zero-injection bus and otherwise $Z_{i}=0$. It is a transhipment node in the system, and usually it is neither a generating unit nor a load bus. Assume index set of zero-injection buses is $\mathscr{Z}=\left\{i_{1}, i_{2}, \cdots, i_{l}, \cdots, i_{L}\right\}$, where there are $L=|\mathscr{Z}|$ zero-injection buses.

Different from the DSP, the power systems have the Ohm's law and the Kirchhoff's current law, which can be used to reduce the number of placed PMUs. We assume the state variable observed on bus $v_{i}$ is the voltage $V_{i}$, and on line $\left(v_{i}, v_{j}\right)$ is the current $I_{i j}$. For a PMU placed on the bus $v_{i}$, it observes the voltage $V_{i}$ and all $I_{i j}$ 's where $v_{j}$ 's are neighbors of $v_{i}$.

- Ohm's law: the current $I_{i j}$ through a conductor between two points is directly proportional to the voltage $V_{i}-V_{j}$ across the two buses $v_{i}, v_{j}$, and inversely proportional to the resistance between them;

- Kirchhoff's current law: at any junction node in an electrical circuit, the sum of currents flowing into that node is equal to the sum of currents flowing out of that node $v_{i}$, i.e., $\sum_{j} I_{i j}=0$. This law is applied to zero-injection buses. In the following, when we mention the property of zero-injection buses, it is actually the Kirchhoff's current law.

Based on these two physical laws, a bus $v_{i}$ of a PSG $G=(V, E)$ is observed by one of the following methods: (a) a PMU is placed on $v_{i}$; (b) its adjacent bus $v_{j}$ is placed with a PMU; and (c) it is one of the buses within $N\left[v_{j}\right]\left(\left(v_{j}, v_{i}\right) \in E\right)$ or $N\left[v_{i}\right]$, while all other buses within the set are observed. Next, based on these three methods, we construct the IP formulation for the bus observability. As pointed out above, if all buses are observed, by Ohm's law, all lines will be observed. Therefore, in the IP model for PPP, the line observability will not be discussed under the situation of complete bus observability.

Before presenting the model, we define the decision variable by a placement vector $x=\left(x_{1}, \cdots, x_{|V|}\right)^{T} \in\{0,1\}^{|V|}$, where $x_{i}=1$ indicates a PMU is placed on bus $v_{i}$, and otherwise $x_{i}=0$. Let $f_{i}$ be the times that bus $v_{i}$ is observed. For the optimal PPP, we consider complete observability that means all buses should be observed. For each pair $(i, j)$ with $v_{i}, v_{j} \in V$, we define $y_{i j} \in\{0,1\}$ such that $y_{i j}=1$ when bus $v_{i}$ is a zero-injection bus and it can provide a coverage for bus $v_{j}$ because of zero-injection bus property, $y_{i j}=0$ if $a_{i j}=0$ or $i \notin \mathscr{Z}$ ( $y$ denotes the vector consisting of $y_{i j}$ for all $i, j)$. Following the IP formulation in [6], the PPP can be solved by the following model:

$$
\begin{array}{ll}
\text { [PPP] } \min _{x, y} & \sum_{i: v_{i} \in V} x_{i} \\
\text { s.t. } & \sum_{j: v_{j} \in N\left[v_{i}\right]} x_{j}+\sum_{j: v_{j} \in N\left[v_{i}\right]} Z_{j} y_{j i} \geq 1, \forall v_{i} \in V \\
& \sum_{j: v_{j} \in N\left[v_{i}\right]} y_{i j}=Z_{i}, \forall v_{i} \in V \\
& y_{i j}=0, \forall i \notin \mathscr{Z}
\end{array}
$$




$$
x_{i}, y_{i j} \in\{0,1\}, \forall v_{i}, v_{j} \in V
$$

where the left-hand-side of constraints (1b) denotes the times $f_{i}$ of observability for bus $v_{i}$,

$$
f_{i}=\sum_{j: v_{j} \in N\left[v_{i}\right]} x_{j}+\sum_{j: v_{j} \in N\left[v_{i}\right]} Z_{j} y_{j i}, \forall v_{i} \in V .
$$

which should be observed at least once. Comparing with the DSP, the first term $\sum_{j: v_{j} \in N\left[v_{i}\right]} x_{j}$ appears in both problems, and it is obtained by method (a) and (b). However, because of the existence of zero-injection bus property, the additional term $\sum_{j: v_{j} \in N\left[v_{i}\right]} Z_{j} y_{j i}$ counts the possible observations. Constraints (1c)-(1d) limit that each zero-injection bus can contribute one time of bus observability within the neighborhood $N\left[v_{i}\right]$. The objective (1a) is to minimize the number of PMUs, and its optimal value is denoted by $\gamma_{p}(G)$. Let $\gamma(G)$ denote the domination number in DSP, which provides an upper bound for the optimal PPP $\gamma_{p}(G)$, i.e., $\gamma_{p}(G) \leq \gamma(G)$.

\subsection{Multistage PMU placement model to maximize observability}

For the multistage PMU placement, we are given the number of stages and the budget limitation for each stage. Assume that there are $T$ stages for PMU placement and it ensures the complete observability at the end of last stage. For stages at $t=$ $1,2, \cdots, T-1$, the placed PMUs will obtain incomplete or partial observability. At the final stage $t=T$, complete observability will be obtained. The budget limits are reflected on the number of PMUs that can be installed by given numbers $n^{1}, n^{2}, \cdots, n^{t}$, $\cdots, n^{T}$, and the total placed PMUs at the end of stage $T$ to ensure complete observability will be $\sum_{t=1}^{T} n^{t}=\gamma_{p}(G)$. The decision variables used in the model for the MPPP include:

- $x_{i}^{t} \in\{0,1\}: x_{i}^{t}=1$ if a PMU is placed on bus $v_{i}$ at the stage $t$ and $x_{i}^{t}=0$ otherwise;

- $y_{i j}^{t} \in\{0,1\}$ : for $a_{i j}=0$ or $i$ is not a zero-injection bus, $y_{i j}^{t}=0$ for all $t=1,2, \cdots, T$; for $i \in \mathscr{Z}, \mathrm{ty}_{i j}^{t}=1$ denotes that the Kirchhoff's current law can provide one time of bus observability when at least $\left|N\left[v_{i}\right]\right|-1$ buses within $N\left[v_{i}\right]$ are observed at stage $t$, and $y_{i j}^{t}=0$ otherwise;

- $z_{i}^{t} \in\{0,1\}: z_{i}^{t}=0$ for all $i \notin \mathscr{Z}, t=1,2, \cdots, T$; for $i \in \mathscr{Z}, z_{i}^{t}=1$ if the zeroinjection bus property on bus $i$ at stage $t$ can be applied and $z_{i}^{t}=0$ otherwise;

- $f_{i}^{t}$ : the times $f_{i}^{t}$ of observability for bus $v_{i}$ at stage $t$ (a nonnegative integer);

- $o_{i}^{t} \in\{0,1\}: o_{i}^{t}=1$ if bus $v_{i}$ is observed at stage $t$ and $o_{i}^{t}=0$ otherwise (it can be decided by $f_{i}^{t}$ ).

Each bus can have at most one PMU and will be placed at some time $t$. Therefore, the constraints for the number of PMUs placed at each stage, and the number for each bus can be expressed as follows, respectively:

$$
\begin{aligned}
& \sum_{i: v_{i} \in V} x_{i}^{t} \leq n^{t}, \forall t=1,2, \cdots, T \\
& \sum_{t=1}^{T} x_{i}^{t} \leq 1, \forall v_{i} \in V
\end{aligned}
$$


The times $f_{i}^{t}$ of observability for bus $v_{i}$ at stage $t$ depends on the PMUs placed on itself and its neighbors, and also the property of zero-injection buses within $N\left[v_{i}\right]$. At stage $t$, the number of PMUs placed on bus $v_{i}$ is $\sum_{t^{\prime}=1}^{t} x_{i}^{t^{\prime}}$. Since during the stage $t(t<$ $T)$, it is incomplete observability and not all buses are observed, the zero-injection bus property can only be used if $z_{i}^{t}=1$, which denotes that zero-injection bus is ready to use at stage $t$. At the last stage $t=T$, all buses should be observed at least once. These constraints can be expressed as follows:

$$
\begin{aligned}
& f_{i}^{t}=\sum_{j: v_{j} \in V} a_{i j}\left(\sum_{t^{\prime}=1}^{t} x_{j}^{t^{\prime}}\right)+\sum_{j: v_{j} \in V} a_{i j} z_{j}^{t} y_{j i}^{t}, \forall v_{i} \in V \\
& \sum_{j: v_{j} \in V} a_{i j} y_{i j}^{t}=z_{i}^{t}, \forall v_{i} \in V \\
& y_{i j}^{t}=0, \forall i, j \text { with } a_{i j}=0 \text { or } i \notin \mathscr{Z}, t=1, \cdots, T \\
& f_{i}^{T} \geq 1, \forall v_{i} \in V
\end{aligned}
$$

The observability $o_{i}^{t} \in\{0,1\}$ is decided by $f_{i}^{t}$ : if $f_{i}^{t} \geq 1, o_{i}^{t}=1$; and if $f_{i}^{t}=0$, $o_{i}^{t}=0$. Assume $\bar{f}$ is a larger number and an upper bound for $f_{i}^{t}$. Thus, the relationship between $o_{i}^{t}$ and $f_{i}^{t}$ can be expressed as follows:

$$
f_{i}^{t} / \bar{f} \leq o_{i}^{t} \leq f_{i}^{t}
$$

The appropriate time $t$ to apply the property of zero-injection bus $i_{l} \in \mathscr{Z}$ is decided by $z_{i_{l}}^{t} \in\{0,1\}$. If all buses except one within the set $N\left[v_{i_{l}}\right]$ are observed at time $t$, the only unobserved bus is also observed by Kirchhoff's current law. If all buses within $N\left[v_{i_{l}}\right]$ are observed at time $t$, then the zero-injection bus property by Kirchhoff's current law can provide one redundancy observability for some bus in $N\left[v_{i_{l}}\right]$. On the other hand, the zero-injection bus property on bus $i_{l} \in \mathscr{Z}$ cannot be applied at time $t$ (i.e., $z_{i_{l}}^{t}=0$ ) if and only if $\sum_{i \in N\left[v_{i_{l}}\right]} o_{i}^{t} \leq\left|N\left[v_{i_{l}}\right]\right|-2$. If the zeroinjection bus property applied at time $t$ and after its application (i.e., $z_{i_{l}}^{t}=1$ ), we have $\sum_{i \in \mathscr{N}_{i_{l}}} o_{i}^{t} \geq\left|N\left[v_{i_{l}}\right]\right|$. This statement can be expressed by

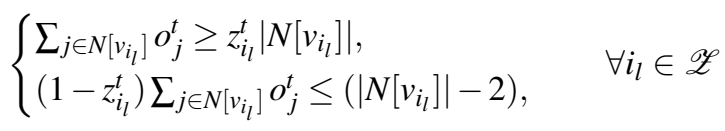

According to the definition of matrix $A,\left|N\left[v_{i_{l}}\right]\right|=\sum_{j: v_{j} \in V} a_{i_{l} j}$. Therefore, we can decide $z_{i_{l}}^{t}$ by the following equivalent constraints:

$$
\begin{array}{r}
\sum_{j: v_{j} \in V} a_{i_{l} j} o_{j}^{t} \geq z_{i_{l}}^{t}\left(\sum_{j: v_{j} \in V} a_{i j}\right), \forall i_{l} \in \mathscr{Z}, \forall t=1,2, \cdots, T \\
\left(1-z_{i_{l}}^{t}\right) \sum_{j: v_{j} \in V} a_{i_{l} j} o_{j}^{t} \leq\left(\sum_{j: v_{j} \in V} a_{i j}-2\right), \forall i_{l} \in \mathscr{Z}, \forall t=1,2, \cdots, T
\end{array}
$$

Additionally, for non zero-injection buses, $z_{i}^{t}$ can be decided by

$$
z_{i}^{t}=0, \forall i \notin \mathscr{Z}, t=1,2, \cdots, T
$$


The installed PMUs cannot be relocated during the next period. Thus the times of observability at stage $t, f_{i}^{t}$ satisfy $f_{i}^{t} \geq f_{i}^{t-1}$, and also $o_{i}^{t} \geq o_{i}^{t-1}, z_{i_{l}}^{t} \geq z_{i_{l}}^{t-1}$ (once the bus is observed $o_{i}^{t-1}=1$, it is always observed; once the zero-injection bus property $z_{i_{l}}^{t-1}=1$ can be used on bus $i_{l}$, it will have this property at next stage).

For each stage $t$, the total times that buses are observed are given by $\sum_{i: v_{i} \in V} f_{i}^{t}$. Therefore, to maximize the overall observability for all stages, the MPPP can be formulated as follows:

$$
\begin{aligned}
\text { [MPPP] } \max _{x, y, z, o, f} & \sum_{t=1}^{T} \sum_{i: v_{i} \in V} f_{i}^{t} \\
\text { s.t. } & (3)-(12) \\
& x_{i}^{t}, y_{i j}^{t}, z_{j}^{t}, o_{i}^{t} \in\{0,1\}, \forall v_{i}, v_{j} \in V, t=1,2, \cdots, T
\end{aligned}
$$

This formulation takes both zero-injection bus property and redundant observation times into account. It helps to make a robust placement schedule with a minimum number of devices. The proposed MIP (13) is nonlinear because of the existence of bilinear terms $y_{j i}^{t} z_{j}^{t}, z_{i_{l}}^{t} o_{j}^{t}$. Since the nonlinear terms are the multiplication of two binary variables, it can be linearized by introducing $u_{i j}^{t}=z_{j}^{t} y_{j i}^{t}, v_{i_{l} j}^{t}=z_{i_{l}}^{t} o_{j}^{t}$, and adding the following constraints

$$
\begin{aligned}
& u_{i j}^{t} \leq z_{j}^{t}, \quad u_{i j}^{t} \leq y_{j i}^{t}, \quad u_{i j}^{t} \geq z_{j}^{t}+y_{j i}^{t}-1, \quad u_{i j}^{t} \geq 0, \quad \forall v_{i}, v_{j} \in V \\
& v_{i_{l} j}^{t} \leq z_{i_{l}}^{t}, \quad v_{i_{l} j}^{t} \leq o_{j}^{t}, \quad v_{i_{l} j}^{t} \geq z_{i_{l}}^{t}+o_{j}^{t}-1, \quad v_{i_{l} j}^{t} \geq 0, \quad \forall i_{l} \in \mathscr{Z}, v_{j} \in V
\end{aligned}
$$

The first two inequalities in (14) ensure that $u_{i j}^{t}$ will be 0 if either $z_{j}^{t}$ or $y_{j i}^{t}$ equals to 0 . The third inequality ensures that $u_{i j}^{t}$ will be 1 if both of $z_{j}^{t}$ and $y_{j i}^{t}$ are 1 . Therefore, the formulation in (13) with additional constraints (14), (15) form an equivalent mixed integer linear program (MILP), which can be solved by CPLEX directly.

Remark 1 When $T=1$, this model solves the following problem: given the number of PMUs which can ensure complete observability, finding the best placement that can maximize the overall bus observability.

\section{Equivalent Network Design Model for PMU Placement}

In this section, we first present the method to obtain an augmented graph for a PSG, and then show how the maximum flow network design model can be used to solve the MPPP. The augmented network design model was first proposed in [13] to solve the single-stage PMU placement problem. Following the idea in [13], we illustrate the augmentation by using the IEEE 14-bus PSG (see Fig. 2), which consists of 14 buses (bus 7 is a zero-injection bus) and 20 transmission lines.

In its augmented graph $N=\left(V^{\prime}, A\right)$ (see Fig. 1), two additional nodes are introduced, including source node $s$ and terminal node $t$. For each bus $v_{i}$ in the original graph, there are two nodes $p_{i}$ and $t_{i}$, two $\operatorname{arcs}\left(p_{i}, t_{i}\right)$ and $\left(t_{i}, t\right)$ in the augmented graph. For each transhipment line $(i, j)$, there are two corresponding nodes $q_{i, j}, r_{i, j}$ and arcs $\left(q_{i, j}, r_{i, j}\right),\left(p_{i}, q_{i, j}\right),\left(p_{j}, q_{i, j}\right),\left(r_{i, j}, t_{i}\right)$ and $\left(r_{i, j}, t_{j}\right)$ in the augmented graph. If a PMU 
is installed on bus $v_{j}$, there is an arc $\left(s, p_{j}\right)$ with capacity $d[i]$ (the cardinality of closed neighborhood of a bus $v_{i}$ ) in the augmented graph. Since bus 7 is a zero-injection bus in the original system, there is a node $z_{7}$, which links to $p_{7}$, and $q_{i, j}$ 's where $i=7$ or $j=7$. In addition, if the zero-injection bus property is applied, there exists an arc $\left(s, z_{7}\right)$. All the arcs in the augmented graph except for $\operatorname{arcs}\left(s, p_{i}\right)$ have one unit flow capacity.

With such construction, we can convert the PPP into a maximum flow network design problem. If a PMU is installed on bus $v_{i}$, there is a flow from $s$ to $p_{i}$. If zeroinjection bus property related to zero-injection bus $v_{j}$ is applied, there is a flow from $s$ to $z_{j}$. If bus $v_{k}$ is observed, then there should be a flow from $t_{k}$ into terminal $t$. If

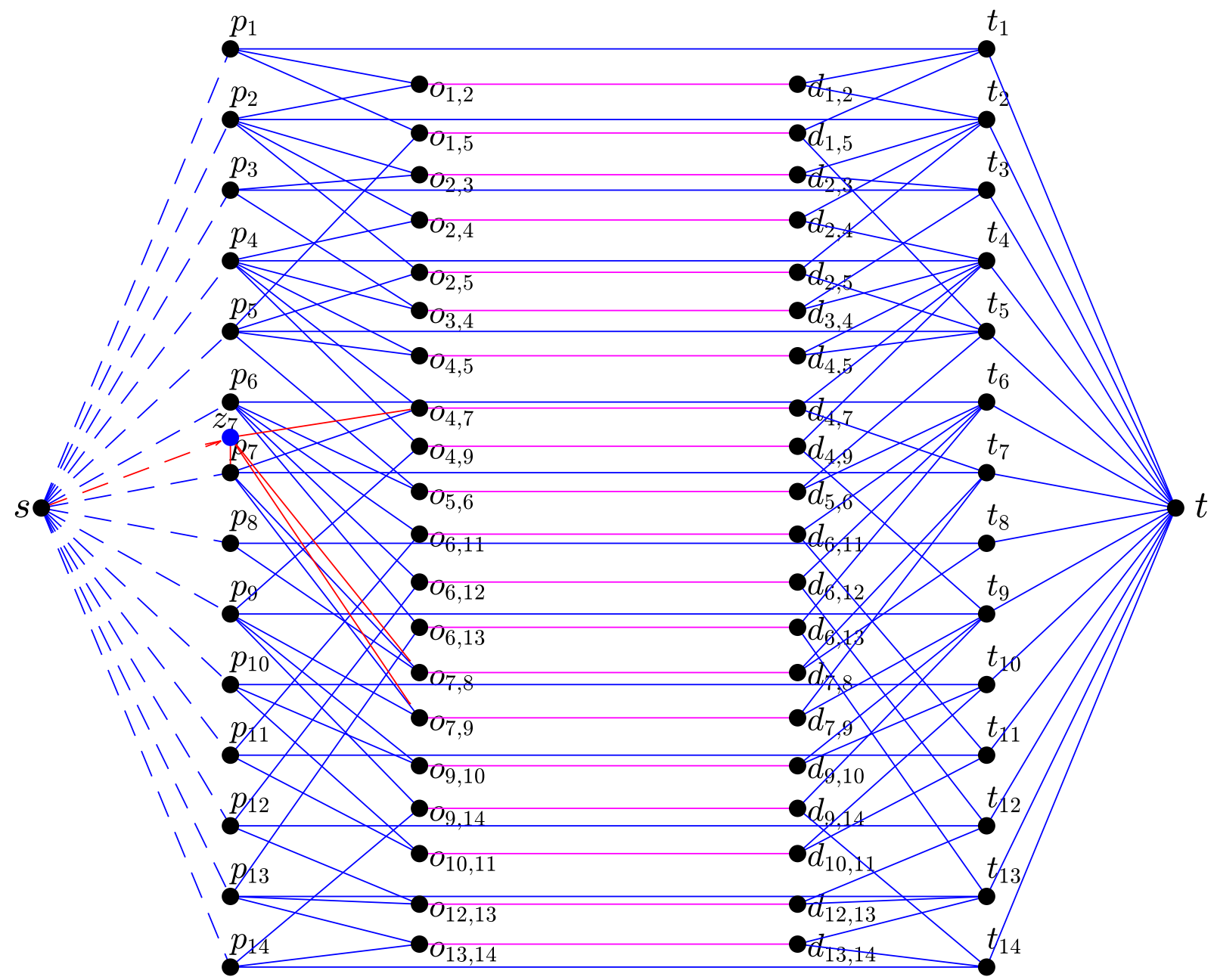

Fig. 1 Augmented Graph for IEEE 14-Bus Power System 
the total number of flows into terminal equals to the number of buses in the system, we can claim that all the buses in the system are observed. For example, if a PMU is installed at bus 1 , a flow up to 3 units is sent from $s$ to $p_{1}$. A unit flow on path $s-p_{1}-t_{1}-t$ means bus 1 can be covered by a PMU installed on itself; a unit flow on path $s-p_{1}-q_{1,2}-r_{1,2}-t_{2}-t$ means bus 2 can be covered by a PMU installed on bus 1; and similarly, a unit flow on path $s-p_{1}-q_{1,5}-r_{1,5}-t_{5}-t$ means bus 5 can be covered by a PMU installed on bus 1 . Furthermore, by applying the zero-injection bus property, one of the buses 4,7,8,9 can be covered by a unit flow sent from $s$ to z7. For example, bus 8 can be observed through zero-injection bus property by a flow on path $s-z_{7}-q_{7,8}-r_{7,8}-t_{8}-t$.

Solving the optimal PPP is equivalent to selecting the minimum number of $p_{i}$ 's (with capacity $d[i]$ 's) to send out flows in order to guarantee that there will be a unit flow from each $t_{i}$ to terminal $t$. For the MPPP, the main difference is that we cannot choose those $p_{i}$ 's at one stage, we need to schedule the selection in $n$ stages due to the budget limits, and the complete observation can only obtained at the last stage. Another difference is that the objective of MPPP is to maximize the flows in the augmented graph instead of minimizing the number of selected $p_{i}$ 's.

For the MPPP, we have different meanings of decision variables under the maximum flow network design model:

$-x_{i}^{t} \in\{0,1\}: x_{i}^{t}=1$ if node $p_{i}$ is selected for sending out flows at stage $t$, and $x_{i}^{t}=0$ otherwise;

- $z_{i_{l}}^{t} \in\{0,1\}: z_{i_{l}}^{t}=1$ if node $z_{i_{l}}$ can be used to send out the flow at stage $t$, and $z_{i_{l}}^{t}=0$ otherwise;

- $y_{i j}^{t}$ : the flow on arc $(i, j)$ at stage $t$.

The overall flows through the system come from two parts, including the flows on $\operatorname{arcs}\left(s, p_{i}\right)$ by selecting nodes $p_{i}\left(\sum_{t=1}^{T} \sum_{i=1}^{n} d[i] \cdot \sum_{t^{\prime}=1}^{t} x_{i}^{t^{\prime}}\right)$ and flows on $\operatorname{arcs}\left(s, z_{i_{l}}\right)$ $\left(\sum_{t=1}^{T} \sum_{i_{l} \in \mathscr{Z}} y_{s z_{i_{l}}}^{t}\right)$ during the whole stages for $t=1,2, \ldots, T$. Since we would like to maximize the flows in the system, the objective function is as follows,

$$
\max \sum_{t=1}^{T} \sum_{i=1}^{n} d[i] \cdot \sum_{t^{\prime}=1}^{t} x_{i}^{t^{\prime}}+\sum_{t=1}^{T} \sum_{i_{l} \in \mathscr{Z}} y_{s z_{i_{l}}}^{t}
$$

At each stage, there is a limitation $n^{t}$ for the number of selected nodes, which is,

$$
\sum_{i=1}^{n} x_{i}^{t} \leq n^{t}, \forall t=1,2, \cdots, T
$$

For each node, it can only be selected once at some stage during the overall periods,

$$
\sum_{t=1}^{T} x_{i}^{t} \leq 1, \forall i \in V
$$

The following three sets of constraints show the capacity for the flow on each arc. If node $p_{i}$ is selected at previous or current stage, then the flow capacity on arc $\left(s, p_{i}\right)$ is $d[i]$. If the node $z_{i_{l}}$ can be used at stage $t$, then the capacity for flow on arc $\left(s, z_{i_{l}}\right)$ 
is $z_{i_{l}}$ since $z_{i_{l}}$ is a binary variable and it obtains value 1 in this case. Otherwise, the capacity of the flow on arc $\left(s, z_{i_{l}}\right)$ is 0 . All the flows on other arcs have the capacity 1.

$$
\begin{aligned}
& 0 \leq y_{s, p_{i}}^{t} \leq d[i] \cdot \sum_{t^{\prime}=1}^{t} x_{i}^{t^{\prime}}, \forall i \in V, \forall t=1, \cdots, T \\
& 0 \leq y_{s, z_{l}}^{t} \leq z_{i_{l}}^{t}, \forall t=1, \cdots, T, \forall i_{l} \in \mathscr{Z} \\
& 0 \leq y_{i j}^{t} \leq 1, \forall(i, j) \in A^{\prime}=A \backslash\left\{\left(s, p_{i}\right),\left(s, z_{i l}\right)\right\}, \forall t=1, \cdots, T
\end{aligned}
$$

For each node in $V^{\prime}$ except for $s$ and $t$, there is a flow balance constraint,

$$
\sum_{j:(i, j) \in A} y_{i j}^{t}-\sum_{j:(j, i) \in A} y_{j i}^{t}=0, \forall i \in V^{\prime} \backslash\{s, t\}, t=1,2, \cdots, T
$$

The following two sets of constraints show the requirements of using nodes $z_{i}$ 's, which have the similar meaning to (10) and (11).

$$
\begin{aligned}
& \sum_{i \in N\left[v_{i_{l}}\right]} y_{t_{i} t}^{t} \geq z_{i_{l}}^{t} d\left[i_{l}\right], \forall i_{l} \in \mathscr{Z}, t=1,2, \cdots, T \\
& \left(1-z_{i_{l}}^{t}\right) \sum_{i \in N\left[v_{i_{l}}\right]} y_{t_{i} t}^{t} \leq d\left[i_{l}\right]-2, \forall i_{l} \in \mathscr{Z}, t=1,2, \cdots, T
\end{aligned}
$$

At last, we need to guarantee the total number of flows into terminal node $t$ at final stage $T$ is equivalent to the total number of buses in the original power system graph, which can be represented as

$$
\sum_{i=1}^{n} y_{t_{i} t}^{T}=|V|
$$

With the network design method, the MPPP problem can be formulated as MIP in the following:

$$
\begin{aligned}
\text { [MPPP-ND] } \quad \max & \sum_{t=1}^{T} \sum_{i=1}^{n} d[i] \cdot \sum_{t^{\prime}=1}^{t} x_{i}^{t^{\prime}}+\sum_{t=1}^{T} \sum_{i_{l} \in \mathscr{Z}} y_{s z_{i_{l}}}^{t} \\
\text { s.t. } & (17)-(25) \\
& x_{i}^{t} \in\{0,1\}, z_{i_{l}}^{t} \in\{0,1\}, \forall v_{i} \in V, i_{l} \in \mathscr{Z}, t=1,2, \cdots, T
\end{aligned}
$$

Similarly, the bilinear term $z_{i_{l}}^{t} \sum_{i \in N\left[v_{i}\right]} y_{t_{i} t}^{t}$ in (24) can be linearized by introducing $u_{i_{l}}^{t}=z_{i_{l}}^{t} \sum_{i \in N\left[v_{\left.i_{l}\right]}\right]} y_{t_{i} t}^{t}$ and adding the following constraints:

$$
\begin{aligned}
& u_{i_{l}}^{t} \leq M z_{i_{l}}^{t}, \quad u_{i_{l}}^{t} \leq \sum_{i \in N\left[v_{i_{l}}\right]} y_{t_{i} t}^{t}, \quad u_{i_{l}}^{t} \geq \sum_{i \in N\left[v_{i_{l}}\right]} y_{t_{i} t}^{t}-\left(1-z_{i_{l}}^{t}\right) M, \\
& u_{i_{l}}^{t} \geq 0, \quad \forall i_{l} \in \mathscr{Z}, t=1,2, \cdots, T
\end{aligned}
$$

where $M$ is an upper bound for $\sum_{i \in N\left[v_{i}\right]} y_{t_{i}}^{t}$. 


\section{Numerical Experiments}

The proposed IP formulations were all implemented in C++ and using CPLEX 12.3 via ILOG Concert Technology 2.9, and all computations were performed on a Linux machine with 4 Intel(R) Xeon(TM) CPU 3.60GHz processors and 32GB RAM. Computational time is reported by CPU seconds.

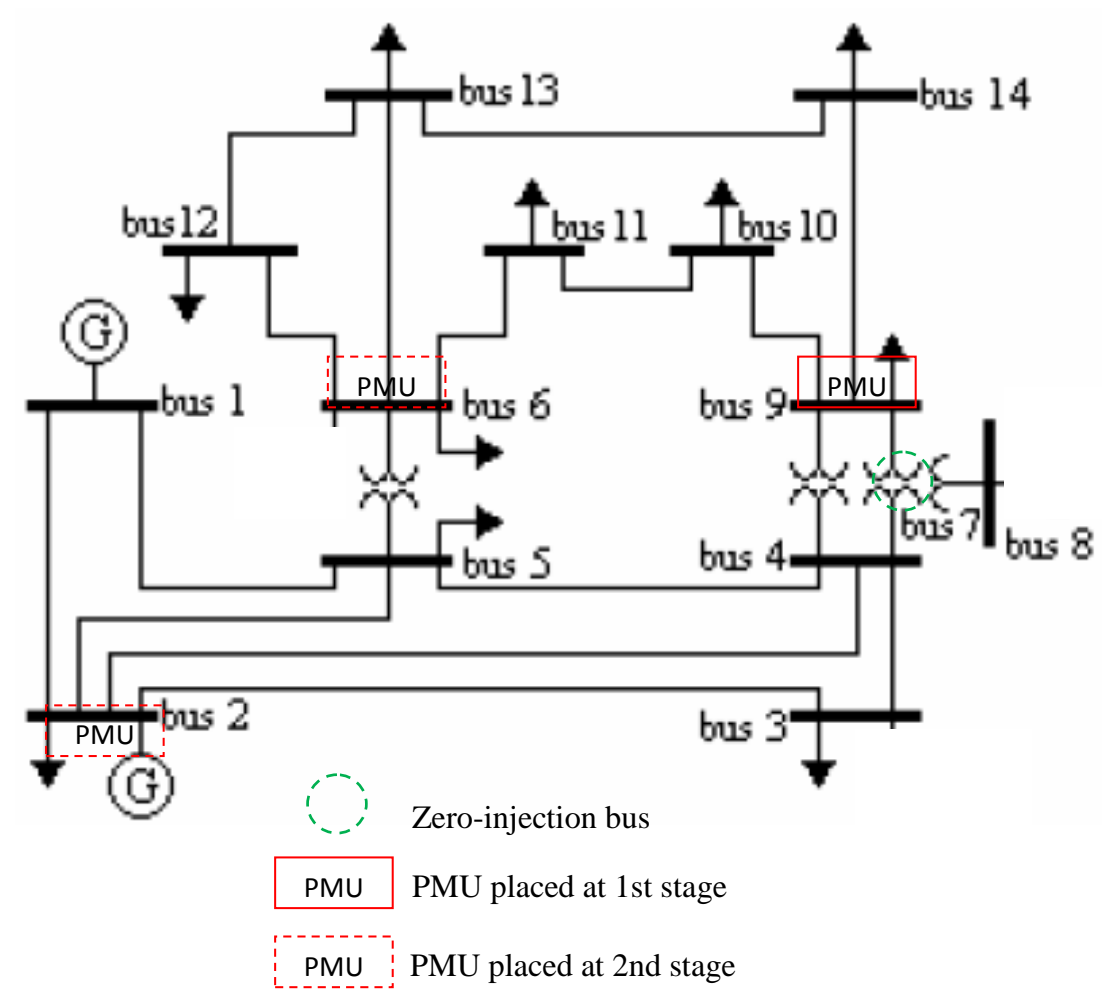

Fig. 2 PMU Placement on IEEE-14-Bus System

First, we are showing the example on IEEE-14-Bus system (see Fig. 2), and by solving the PPP, there are at least 3 PMUs needed (i.e., $\gamma_{p}(G)=3$ ). There is one zero-injection bus in this system (bus 7). Assume $T=2, n^{1}=1, n^{2}=2$. At the first stage, if the available one PMU is placed on bus 9, bus 9 will be observed directly; buses 4,7,10,14 will be observed by Ohm's law; and by Kirchhoff's current law on bus 7 (within $N\left[v_{7}\right]$, buses $4,7,9$ are already observed), bus 8 is also observed. Thus, the total number of observed buses at this stage is 6 . Any other placement at this stage will not have the result that 6 buses can be observed. At the 2nd stage, two other PMUs will be placed on buses 2,6 to ensure complete bus observability and maximum overall observability. 
In Table 1, we test 6 systems, and the optimal solutions for models DSP, VCP and PPP are all shown with the computational time. Normally, we have the results that $\gamma_{p}(G) \leq \gamma(G) \leq \tau(G)$. All of them can be solved within 1 second.

Table 1 Computational Results by Models DSP, VCP, PPP

\begin{tabular}{|c|c|c|c|c|c|c|c|c|c|}
\hline \multirow[b]{2}{*}{ Name } & \multirow{2}{*}{$\begin{array}{l}\text { System } \\
|V|\end{array}$} & \multirow[b]{2}{*}{$|E|$} & \multirow[b]{2}{*}{$\sum_{i} Z_{i}$} & \multirow{2}{*}{$\begin{array}{l}\text { DSP } \\
\gamma(G)\end{array}$} & \multirow{2}{*}{$\begin{array}{l}\mathrm{VCP} \\
\tau(G)\end{array}$} & \multirow{2}{*}{$\begin{array}{r}\text { PPP } \\
\gamma_{p}(G)\end{array}$} & \multicolumn{3}{|c|}{ CPU Seconds } \\
\hline & & & & & & & DSP & $\mathrm{VCP}$ & PPP \\
\hline IEEE-14-Bus & 14 & 20 & 1 & 4 & 8 & 3 & 0 & 0 & 0 \\
\hline IEEE-30-Bus & 30 & 41 & 6 & 10 & 16 & 7 & 0 & 0.01 & 0 \\
\hline IEEE-57-Bus & 57 & $80(2)$ & 15 & 17 & 30 & 11 & 0.01 & 0.01 & 0.01 \\
\hline RTS-96 & 73 & $120(12)$ & 22 & 20 & 39 & 14 & 0.01 & 0.01 & 0.03 \\
\hline IEEE-118-Bus & 118 & $186(7)$ & 10 & 32 & 61 & 28 & 0.01 & 0.03 & 0.04 \\
\hline IEEE-300-Bus & 300 & $411(2)$ & 65 & 87 & 136 & 68 & 0.01 & 0.34 & 0.41 \\
\hline
\end{tabular}

Note: The column $|E|$ with () denotes the number of lines with the number of pairs for parallel lines. The column $\sum_{i} Z_{i}$ denotes the number of zero-injection buses.

In Table 2, for different values of $T$, we assume that the number $n^{t}$ of PMUs at each stage is around $\gamma_{p}(G) / N$. Regarding different number of stages, the PMU placement schedule is shown in this table. Different from results in $[8,31]$, which did not consider zero-injection buses for multistage, here the use of zero-injection bus property can maximize the observability at the very early stage. We present results based on models MPPP and MPPP-ND in the table. From the result, we can see, when it comes to a larger system with more stages, MPPP-ND is more efficient than MPPP model.

Next, we compare our results with paper [32], where optimization method was used but constraints should be added case by case. Here, we can see that our method is more explicit and can be extended for solving any system. For multistage PMU placement, [32] only studied the IEEE-30-Bus, IEEE-57-Bus and IEEE118-Bus systems, all of which we are comparing in Table 3. According to the figure for IEEE118-Bus system [5], the bus 33,35 are unobserved by the results in [32]. To obtain the observability $159(=118+41)$, one additional PMU should be added at bus 37 .

\section{Conclusions}

In this paper, we present a mixed integer programming formulations for multistage PMU placement problem by considering maximizing bus observability and also an equivalent maximum flow network design model during all stages. By solving the MIP formulations, we present a multistage scheduling for PMU installations in a long-term time period where each period has a budget limit. Additionally, since the total number of PMUs being placed is limited by the solution of the optimal PMU placement problem, our models achieve best placement which obtains most measurements for observability at each stage. The equivalent network design model shows its computational benefits. With consideration of random failures of PMUs, buses and transmission lines, the future work includes study of robust placement of PMUs and operations of them under different scenarios of contingencies. 
Table 2 Computational Results by the Model MPPP and MPPP-ND

\begin{tabular}{|c|c|c|c|c|c|c|c|c|c|c|}
\hline Systems & $\gamma_{p}(G)$ & $T$ & $t$ & $n^{t}$ & Obj. & Stage Obs. & Placement & Used 0-Bus & Time (MPPP) & Time (MPPP-ND) \\
\hline \multirow{8}{*}{ IEEE-14-Bus } & \multirow[t]{8}{*}{3} & 1 & $t=1$ & 3 & 16 & $16(16)$ & $2,6,9$ & 7 & 0 & 0 \\
\hline & & 2 & $t=1$ & 1 & 22 & $6(6)$ & 9 & 7 & 0 & 0 \\
\hline & & & $t=2$ & 2 & & $16(14)$ & 2,6 & 7 & & \\
\hline & & 2 & $t=1$ & 2 & 27 & $11(10)$ & 2,9 & 7 & 0 & 0 \\
\hline & & & $t=2$ & 1 & & $16(14)$ & 6 & 7 & & \\
\hline & & 3 & $t=1$ & 1 & 33 & $6(6)$ & 9 & 7 & 0.01 & 0.02 \\
\hline & & & $t=2$ & 1 & & $11(10)$ & 6 & 7 & & \\
\hline & & & $t=3$ & 1 & & $16(14)$ & 2 & 7 & & \\
\hline \multirow[t]{13}{*}{ IEEE-30-Bus } & \multirow[t]{13}{*}{7} & 1 & $t=1$ & 7 & 42 & $42(30)$ & $2,4,10,12,15,18,27$ & $6,9,22,25,27,28$ & 0.01 & 0.05 \\
\hline & & 2 & $t=1$ & 3 & 65 & $23(21)$ & $2,10,27$ & $6,9,22,25,27,28$ & 0.04 & 0.13 \\
\hline & & & $t=2$ & 4 & & $42(30)$ & $4,12,15,18$ & $6,9,22,25,27,28$ & & \\
\hline & & 3 & $t=1$ & 2 & 88 & $17(16)$ & 10,27 & $9,22,25,27,28$ & 0.09 & 0.43 \\
\hline & & & $t=2$ & 2 & & $29(25)$ & 4,12 & $6,9,22,25,27,28$ & & \\
\hline & & & $t=3$ & 3 & & $42(30)$ & $2,15,18$ & $6,9,22,25,27,28$ & & \\
\hline & & 3 & $t=1$ & 2 & 93 & $17(16)$ & 10,27 & $9,22,25,27,28$ & 0.1 & 0.26 \\
\hline & & & $t=2$ & 3 & & $34(27)$ & $2,4,12$ & $6,9,22,25,27,28$ & & \\
\hline & & & $t=3$ & 2 & & $42(30)$ & 15,18 & $6,9,22,25,27,28$ & & \\
\hline & & 4 & $t=1$ & $\frac{2}{2}$ & 127 & $\frac{42(10)}{17(16)}$ & $\frac{10,10}{10,27}$ & $\frac{0,9,2,2,25,2,20}{9,22,25,28}$ & 0.14 & 0.44 \\
\hline & & & $t=2$ & 2 & & $29(26)$ & 2,12 & $6,9,22,25,27,28$ & & \\
\hline & & & $t=3$ & 2 & & $39(29)$ & 4,15 & $6,9,22,25,27,28$ & & \\
\hline & & & $t=4$ & 1 & & $42(30)$ & 19 & $6,9,22,25,27,28$ & & \\
\hline \multirow[t]{6}{*}{ IEEE-57-Bus } & \multirow[t]{6}{*}{11} & 1 & $t=1$ & 11 & 63 & $63(57)$ & $1,6,13,19,25,29,32,38,41,51,54$ & all 15 used & 0.02 & 0.15 \\
\hline & & 2 & $t=1$ & 6 & 106 & $43(40)$ & $1,6,13,19,38,56$ & 12 of 15 used & 0.38 & 1.13 \\
\hline & & & $t=2$ & 5 & & $63(57)$ & $25,29,32,51,54$ & all 15 used & & \\
\hline & & 3 & $t=1$ & 4 & 148 & $32(30)$ & $6,13,38,56$ & 9 of 15 used & 0.87 & 2.04 \\
\hline & & & $t=2$ & 4 & & $53(49)$ & $1,19,25,32$ & all 15 used & & \\
\hline & & & $t=3$ & 3 & & $63(57)$ & $29,51,54$ & all 15 used & & \\
\hline \multirow[t]{6}{*}{ RTS-96 } & \multirow[t]{6}{*}{14} & 1 & $t=1$ & 14 & 92 & $92(73)$ & $1,9,10,16,26,27,32,34,40,50,56,57,58,64$ & all 22 used & 0.02 & 0.05 \\
\hline & & 2 & $t=1$ & 7 & 149 & $57(51)$ & $9,10,16,40,57,58,64$ & 18 of 22 used & 0.94 & 5.81 \\
\hline & & & $t=2$ & 7 & & $92(73)$ & $1,26,27,32,34,50,56$ & all 22 used & & \\
\hline & & 3 & $t=1$ & 5 & 208 & $40(37)$ & $9,16,57,58,64$ & 12 of 22 used & 5.75 & 2.6 \\
\hline & & & $t=2$ & 5 & & $76(67)$ & $10,27,34,40,49$ & all 22 used & & \\
\hline & & & $t=3$ & 4 & & $92(73)$ & $2,26,32,56$ & all 22 used & & \\
\hline \multirow[t]{9}{*}{ IEEE-118-Bus } & \multirow[t]{9}{*}{28} & 1 & $t=1$ & 28 & 157 & $157(118)$ & $\begin{array}{l}3,8,11,12,17,21,27,31,32,34,37,40,45,49,52 \\
56,62,72,75,77,80,85,86,90,94,102,105,110\end{array}$ & 10 used & 0.06 & 0.69 \\
\hline & & 2 & $t=1$ & 14 & 257 & $100(85)$ & $8,11,12,17,37,49,56,62,75,77,80,85,94,105$ & 9 of 10 used & 0.47 & 1.69 \\
\hline & & & $t=2$ & 14 & & $157(118)$ & $3,21,27,31,32,34,40,45,52,72,86,90,102,110$ & 10 used & & \\
\hline & & 3 & $t=1$ & 10 & 359 & $76(65)$ & $8,11,12,17,37,49,56,62,77,80$ & 8 of 10 used & 1.24 & 1.37 \\
\hline & & & $t=2$ & 9 & & $126(100)$ & $27,32,34,40,75,85,94,105,110$ & 9 of 10 used & & \\
\hline & & & $t=3$ & 9 & & $157(118)$ & $3,21,31,45,52,72,86,90,102$ & 10 used & & \\
\hline & & 3 & $t=1$ & 9 & 353 & $70(62)$ & $8,12,17,37,49,56,62,77,80$ & 8 of 10 used & 1.47 & 1.76 \\
\hline & & & $t=2$ & 10 & & $126(100)$ & $11,27,32,34,75,85,94,105,110$ & 9 of 10 used & & \\
\hline & & & $t=3$ & 9 & & $157(118)$ & $3,21,31,45,52,72,86,90,102$ & 10 used & & \\
\hline \multirow[t]{18}{*}{ IEEE-300-Bus } & \multirow[t]{18}{*}{68} & 1 & $t=1$ & 68 & 409 & $409(300)$ & $\begin{array}{l}1,2,3,11,15,17,20,22,23,25,27,37,38,43,48,49,53,55, \\
58,59,60,64,79,85,86,88,89,93,98,99,101,105,116, \\
118,119,124,132,135,141,154,157,163,167,169,177, \\
184,189,190,193,196,202,204,208,210,211,213,216, \\
217,224,228,267,268,269,270,272,274,276,294\end{array}$ & 65 used & 0.34 & 3.04 \\
\hline & & 3 & $t=1$ & 23 & 939 & $203(174)$ & $3,11,15,20,23,27,38,64,79,89,99,105,116,119$ & 48 of 66 used & 133.13 & 19 \\
\hline & & & $t=2$ & 23 & & $327(255)$ & $\begin{array}{l}122,177,189,190,210,216,268,270,272 \\
2,22,37,43,48,49,53,59,60,86,98,101\end{array}$ & 64 used & & \\
\hline & & & & & & & $141,152,167,169,184,196,213,224,269,274,276$ & & & \\
\hline & & & $t=3$ & 22 & & $409(300)$ & $\begin{array}{l}1,17,25,55,58,85,88,93,118,132,135,157 \\
163,193,202,204,208,211,217,228,267,294\end{array}$ & 65 used & & \\
\hline & & 5 & $t=1$ & 14 & 1458 & $141(130)$ & $3,27,38,64,99,105,116,119,122,190,210,216,268,270$ & 38 of 66 used & 407.59 & 68.07 \\
\hline & & & $t=2$ & 14 & & $233(198)$ & $2,11,15,20,23,49,59,79,89,177,189,196,224,272$ & 53 used & & \\
\hline & & & $t=3$ & 14 & & $307(244)$ & $37,43,48,53,60,86,98,101,141,152,184,213,269,276$ & 62 used & & \\
\hline & & & $t=4$ & 14 & & $368(275)$ & $1,22,55,85,88,118,157,167,169,193,204,208,217,274$ & 64 used & & \\
\hline & & & $t=5$ & 12 & & $409(300)$ & $17,25,58,93,132,135,163,202,211,228,267,294$ & 65 used & & \\
\hline & & 8 & $t=1$ & 9 & 2235 & $98(96)$ & $3,27,38,64,105,122,190,216,268$ & 30 of 65 used & 1411.4 & 182.26 \\
\hline & & & $t=2$ & 9 & & $171(155)$ & $15,23,79,99,116,119,189,210,270$ & 44 used & & \\
\hline & & & $t=3$ & 9 & & $227(193)$ & $11,20,89,101,152,177,196,224,272$ & 52 used & & \\
\hline & & & $t=4$ & 9 & & $277(232)$ & $2,37,43,49,53,59,141,184,276$ & 62 used & & \\
\hline & & & $t=5$ & 8 & & $317(252)$ & $22,48,60,86,157,167,213,269$ & 62 used & & \\
\hline & & & $t=6$ & 8 & & $352(268)$ & $55,93,98,118,169,193,217,274$ & 64 used & & \\
\hline & & & $t=7$ & 8 & & $384(285)$ & $1,25,85,88,132,104,208,267$ & 64 used & & \\
\hline & & & $t=8$ & 8 & & $409(300)$ & $17,58,135,163,202,211,228,296$ & 65 used & & \\
\hline
\end{tabular}

Note: The obj. denotes the value of the objective function in the MPPP model; Stage Obs. with () denotes $\sum_{i} f_{i}^{t}$ for stage $t$, and number in the parenthesis is number of observed buses for that stage; Used 0-Bus denotes the buses on which zero-injection bus property is used at that stage. 
Table 3 Results Comparison with [32] for Maximized Observability

\begin{tabular}{|c|c|c|c|c|c|c|c|c|c|}
\hline \multirow[b]{2}{*}{ System } & \multirow[b]{2}{*}{$T$} & \multirow[b]{2}{*}{$t$} & \multirow[b]{2}{*}{$n^{t}$} & \multicolumn{3}{|c|}{ Results from [32] } & \multicolumn{3}{|c|}{ Results from Table 2} \\
\hline & & & & Bus Obs. & Placement & \# 0-Bus & Bus Obs. & Placement & $\# 0$-Bus \\
\hline IEEE-30-Bus & 1 & & 7 & 41 & $2,4,10,12,15,20,27$ & 5 & 42 & $2,4,10,12,15,19,27$ & 6 \\
\hline IEEE-57-Bus & 1 & & 11 & 61 & $1,4,13,20,25,29,32,38,51,54,56$ & 13 & 63 & $1,6,13,19,25,29,32,38,41,51,54$ & 15 \\
\hline IEEE-118-Bus & 1 & & 28 & 159(x) & $\begin{array}{l}2,9,11,12,17,21,27,31,32,34,40, \\
45,49,52,56,62,65,72,75,77,80, \\
85,87,90,94,101,105,110\end{array}$ & 9 & 157 & $\begin{array}{l}3,8,11,12,17,21,27,31,32,34,37 \\
40,45,49,52,56,62,72,75,77,80 \\
85,86,90,94,102,105,110\end{array}$ & 10 \\
\hline \multirow[t]{3}{*}{ IEEE-30-Bus } & 3 & $t=1$ & 2 & 17 & 10,27 & 5 & 17 & 10,27 & 5 \\
\hline & & $t=2$ & 3 & 34 & $2,4,12$ & 6 & 34 & $2,4,12$ & 6 \\
\hline & & $t=3$ & 2 & 42 & 15,20 & 6 & 42 & 15,19 & 6 \\
\hline \multirow[t]{3}{*}{ IEEE-57-Bus } & 3 & $t=1$ & 4 & 27 & $1,4,13,38$ & 4 & 32 & $6,13,38,56$ & 9 \\
\hline & & $t=2$ & 4 & 50 & $25,29,32,56$ & 11 & 53 & $1,19,25,32$ & 15 \\
\hline & & $t=3$ & 3 & 63 & $20,51,54$ & 15 & 63 & $29,51,54$ & 15 \\
\hline \multirow[t]{3}{*}{ IEEE-118-Bus } & 3 & $t=1$ & 9 & 68 & $12,17,49,56,65,75,77,80,85$ & 5 & 70 & $8,12,17,37,49,56,62,77,80$ & 8 \\
\hline & & $t=2$ & 10 & 119 & $9,11,31,34,40,45,62,94,105,110$ & 8 & 126 & $11,27,32,34,40,75,85,94,105,110$ & 9 \\
\hline & & $t=3$ & 9 & 151 & $2,21,27,32,52,72,87,90,101$ & 9 & 157 & $3,21,31,45,52,72,86,90,102$ & 10 \\
\hline
\end{tabular}

Note: The Bus Obs. denotes the value of the objective function in the MPPP model; \# 0-Bus denotes the number of zero-injection buses on which the zero-injection bus property is used at that stage.

\section{References}

1. P. Overholt, D. Ortiz, \& A. Silverstein. "Synchrophasor technology and the doe: Exciting opportunities lie ahead in development and deployment." IEEE Power and Energy Magazine, vol 13 (5), pp. 14-17, 2015.

2. B. Xu, A. Abur, "Observability analysis and measurement placement for systems with PMUs", Proceedings of 2004 IEEE PES Conference and Exposition, vol. 2, pp. 943-946, 2004.

3. B. Gou, "Generalized integer linear programming formulation for optimal PMU placement", IEEE Trans. Power Syst. 23 (3), pp. 1099-1104, 2008.

4. B. Gou, "Optimal placement of PMUs by integer linear programming", IEEE Trans. Power Syst. 23 (3), pp. 1525-1526, 2008.

5. M. Korkah, "Strategic and robust deployment of synchronized phasor measurement units with restricted channel capacity", MS thesis, Northeastern University, 2010.

6. N. Fan, J.P. Watson, "Solving the connected dominating set problem and power dominating set problem by integer programming", Combinatorial Optimization and Applications, pp. 371-383, 2012.

7. F. Aminifar, M. Fotuhi-Firuzabad, M. Shahidehpour, A. Khodaei, "Observability enhancement by optimal placement considering random power system outages", Energy Systems, vol. 2, pp. 25-65, 2011.

8. D. Dua, S. Dambhare, R.K. Gajbhiye, S.A.Soman, "Optimal multistage scheduling of PMU placement: An ILP approach”, IEEE Trans. on Power Delivery, vol. 23, pp. 1812-1820, 2008.

9. S. Chakrabarti, E.Kyriakides, D.G. Eliades, "Placement of synchronized measurements for power system observability", IEEE Trans. Power Deliv. 24 (1), pp. 12-19, 2009.

10. F. Aminifar, A. Khodaei, M. Fotuhi-Firuzabad, M. Shahidehpour, "Contingency-constrained PMU placement in power networks", IEEE Trans. on Power Systems, vol. 25, pp. 516-523, 2010.

11. A. Enshaee, R.A. Hooshmand, \& F. H. Fesharaki. " A new method for optimal placement of phasor measurement units to maintain full network observability under various contingencies", Electric Power Systems Research, vol. 89, pp. 1-10, 2012.

12. F. Rashidi, et al. "Optimal placement of PMUs with limited number of channels for complete topological observability of power systems under various contingencies", International Journal of Electrical Power \& Energy Systems, vol. 67, pp. 125-137, 2015.

13. R. L. Chen, J. Ruthruff. "A scalable decomposition algorithm for PMU placement under multiplefailure contingencies." PES General Meeting Conference \& Exposition, pp. 1-5, IEEE, 2014.

14. N. M. Manousakis, G. N. Korres, \& P. S. Georgilakis. "Taxonomy of PMU placement methodologies", IEEE Transactions on Power Systems, vol. 27 (2), pp. 1070-1077, 2012.

15. R. Babu, B. Bhattacharyya. "Optimal allocation of phasor measurement unit for full observability of the connected power network", International Journal of Electrical Power \& Energy Systems, vol. 79, pp. 89-97, 2016.

16. N. Fan, J.P. Watson, "On integer programming models for the multi-channel PMU placement problem and their solution", Energy Systems, Vol. 6 (1), pp. 1-19, 2015.

17. X. Zhu, et al. "New dominating sets in social networks." Journal of Global Optimization, vol. 48 (4), pp. 633-642, 2010. 
18. S. Butenko, X. Cheng, C. A. Oliveira, \& P. M. Pardalos. "A new heuristic for the minimum connected dominating set problem on ad hoc wireless networks." Recent developments in cooperative control and optimization, pp. 61-73. Springer US, 2004.

19. M. Min, et al."Improving construction for connected dominating set with Steiner tree in wireless sensor networks." Journal of Global Optimization, vol. 35 (1), pp. 111-119, 2006.

20. Z. Zhang, et al. "Minimum vertex cover in ball graphs through local search." Journal of Global Optimization, vol. 59 (2-3), pp. 663-671, 2014.

21. X. Liu, et al. "PTAS for the minimum k-path connected vertex cover problem in unit disk graphs." Journal of Global Optimization, vol. 56 (2), pp. 449-458, 2013.

22. T.W. Haynes, S.M. Hedetniemi, S.T. Hedetniemi, M. A. Henning, "Domination in graphs applied to electric power networks", SIAM J. Discrete Math., vol. 15, pp. 519-529, 2002.

23. M. Zhao, L. Kang, "Power domination in planar graphs with small diameter", Journal of Shanghai University (English Edition), vol. 11 (3), pp. 218-222, 2007.

24. P. Dorbec, M. Mollard, S. Klavzar, S. Spacapan, "Power domination in product graphs", SIAM J. Discret. Math. vol. 22 (2), pp. 554-567, 2008.

25. R. Barrera, D. Ferrero, "Power domination in cylinders, tori, and generalized Petersen graphs", Networks, 58, pp. 43-49, 2011.

26. G. Xu, L. Kang, "On the power domination number of the generalized Petersen graphs", Journal of Combinatorial Optimization, vol. 22 (2), pp. 282-291, 2011.

27. D.J. Bruent, L.S. Health, “The PMU placement problem”, SIAM J. Discrete Math., vol. 19, pp. 744 761, 2005.

28. US Department of Energy, Electricity Delivery \& Energy Reliability, Factors affecting PMU installation costs, Smart Grid Investment Grant Program, October 2014. Available at https: //www. smartgrid.gov/files/PMU-cost-study-final-10162014_1.pdf (accessed on Sep 5, 2017).

29. J.R. Altman, "A practical comprehensive approach to PMU placement for full observability", MS thesis, Virginia Polytechnic Institute and State University, 2007.

30. R. Sodhi, S.C. Srivastava, S.N. Singh, "Multi-criteria decision-making approach for multistage optimal placement of phasor measurement unit", IET Gener. Transm. Distrib., vol. 5 (2), pp. 181-190, 2011.

31. F. Aminifar, M. Fotuhi-Firuzabad, M. Shahidehpour, A. Khodaei, "Probabilistic multistage PMU placement in electric power systems", IEEE Trans. on Power Delivery, vol. 26(2), pp. 841-849, April 2011.

32. S.M. Mahaei, M.T. Hagh, "Minimizing the number of PMUs and their optimal placement in power systems", Elec. Power Syst. Res., vol. 83, pp. 66-72, 2012

33. S. E. Razavi, H. Falaghi, \& M. Ramezani."A new integer linear programming approach for multi-stage PMU placement.” Smart Grid Conference (SGC), pp. 119-124, IEEE, 2013.

34. M.H. Wen, J. Xu, \&V.O. Li. "Optimal multistage PMU placement for wide-area monitoring." IEEE Transactions on Power Systems, vol. 28 (4), pp. 4134-4143, 2013. 